\title{
Memory-Guided Learning: CA1 and CA3 Neuronal Ensembles Differentially Encode the Commonalities and Differences between Situations
}

\author{
Amir S. Bahar, Prasad R. Shirvalkar, and Matthew L. Shapiro \\ Fishberg Department of Neuroscience and Alfred B. and Gudrun J. Kastor Neurobiology of Aging Laboratories, Mount Sinai School of Medicine, New York, \\ New York 10029-6574
}

\begin{abstract}
Memory influences learning, but how neural signals support such transfer are unknown. To investigate these mechanisms, we trained rats to perform a standard spatial memory task in a plus maze and tested how training affected learning and neural coding in two new task variants. A switch task exchanged the start and goal locations in the same environment, whereas, an altered environment task contained unfamiliar local and distal cues. Learning was facilitated in both variants compared with the acquisition of the standard task. In the switch task, performance was largely maintained, and was accompanied by immediate and stable place-field remapping. Place-field maps in CA1 were anticorrelated in the standard and switch sessions, and the anticorrelation covaried with switch performance. Simultaneously, CA3 maps were uncorrelated overall in the standard and switch, though many CA3 cells had fields in shifted locations in the same maze arms. In the altered environment, performance was initially impaired, and place fields changed dynamically. CA1 fields were initially unstable, and their stabilization correlated with improving performance. Most CA3 cells, however, stopped firing on the maze in the altered environment, even as the same cells maintained prominent fields in standard sessions recorded before and after. CA1 and CA3 place fields thus revealed different coding dynamics that correlated with both learning and memory performance. Together, CA1 and CA3 ensembles represented the similarities and differences between new and familiar situations through concurrent rate and place remapping.
\end{abstract}

\section{Introduction}

In adults, new experiences occur in the context of established memories that influence the content and speed of learning (Thorndike, 1919). Positive transfer, for example, through learning sets or mental schemas, generalizes relevant information across similar situations (Bartlett, 1932; Harlow, 1949). Negative transfer requires discriminating previously irrelevant features or suppressing responses to conflicting signals. The neural mechanisms by which memory influences learning are largely unknown.

The dynamic coding properties of hippocampal cells provide an excellent model for investigating how established neuronal representations respond during learning. Memory for spatial episodes requires the hippocampus, a brain structure whose neurons fire in specific locations (place fields), as animals move through familiar environments. The formation, maintenance, and reliable activation of place fields suggest plausible candidate mechanisms for learning, storing, and retrieving spatial memo-

Received April 1, 2011; revised May 13, 2011; accepted July 6, 2011.

Author contributions: A.S.B. and M.L.S. designed research; A.S.B. performed research; A.S.B., P.R.S., and M.L.S. analyzed data; A.S.B., P.R.S., and M.L.S. wrote the paper.

This work was supported by the Mount Sinai School of Medicine, National Institutes of Health Grants MH065658 and MH073689, and a 2008 NARSAD (National Alliance for Research on Schizophrenia and Depression) Young Investigator award to A.S.B. We thank Maojuan Zhang for technical assistance. Kimberly Kwei, Justin Riceberg, Pamela Kennedy, and Jim Knierim for their comments on the manuscript.

Correspondence should be addressed to Dr. Matthew L. Shapiro, Mount Sinai School of Medicine, One Gustave Levy Place, Box 1065, New York, NY 10029. E-mail: matthew.shapiro@mssm.edu.

DOI:10.1523/JNEUROSCI.1671-11.2011

Copyright $\odot 2011$ the authors $\quad 0270-6474 / 11 / 3112270-12 \$ 15.00 / 0$ ries (O'Keefe and Nadel, 1978). When rats explore an unfamiliar environment, new and stable fields form rapidly (Wilson and McNaughton, 1993), and reflect behavioral structures, for example, distinguishing movement direction when rats make different trajectories through the same places (McNaughton et al., 1983). Place fields respond dynamically to changes to environmental features and task contingencies, and the response varies across manipulations (Muller and Kubie, 1987; Fenton et al., 2000). In global remapping, the active population and the spatial distribution of firing rates are unpredictable from one situation to the other (Jeffery et al., 2003); in rate remapping, the same population is active in the same locations, but the cells fire with different rates (Leutgeb et al., 2005). In partial remapping, the fields of some cells are maintained while others are not (Shapiro et al., 1997; Knierim, 2002). Though different memory functions have been attributed to different remapping patterns (Leutgeb and Leutgeb, 2007), few experiments have assessed these patterns during learning and memory performance.

We recorded ensembles of CA1 and CA3 neurons as rats learned hippocampus-dependent memory tasks (Ferbinteanu and Shapiro, 2003). Rats were trained to perform a standard (STD) matching-to-place task with serial reversals on a plus maze (Fig. 1). Each rat was placed in either the North or South start arm, and could find food at the end of either the East or West goal arm. Later, the opposite goal was rewarded between alternating trial blocks. After reaching asymptotic performance in the STD task, the rats were presented with two task variants that followed the same rules, but required different discriminations. In a switch 
a Pre-training $\longrightarrow$ Electrode implant $\longrightarrow$ Recovery $\longrightarrow$ Retraining $\downarrow$

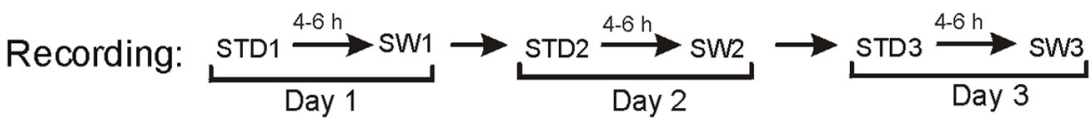

b

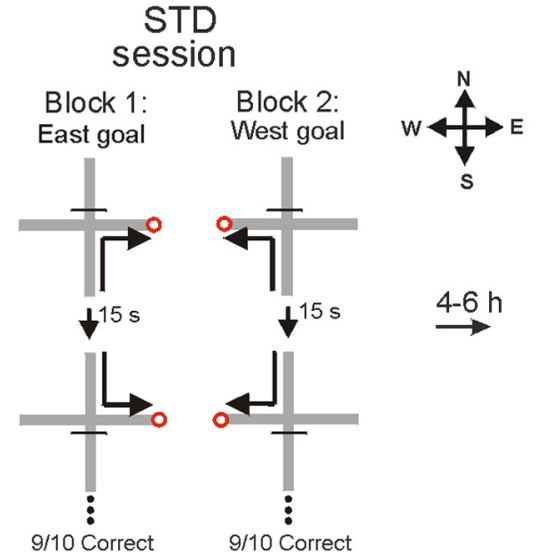

SW session Block 1: Block 2: South goal North goal

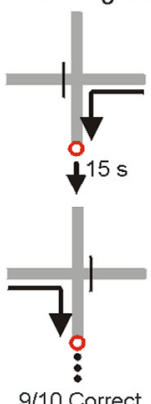

C

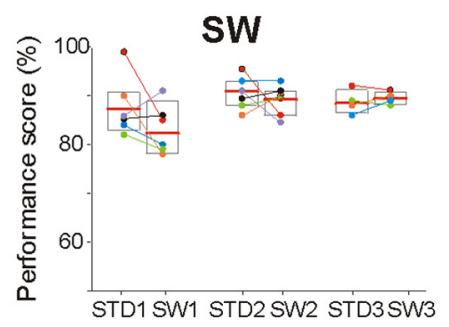

e

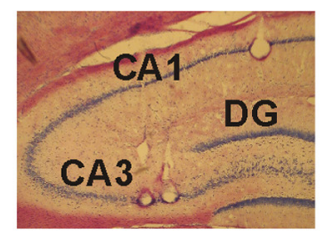

f

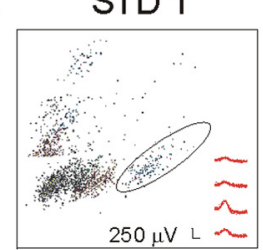

d

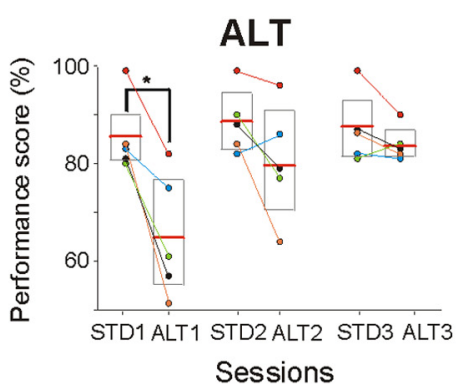

Figure 1. Experimental design, performance, and recording stability. $\boldsymbol{a}$, The recording protocols of the SW and ALT tasks (data not shown) were identical. $\boldsymbol{b}$, Rats were trained in a spatial win-stay task with serial reversals on a plus maze. In the familiar STD session rats performed 10 trials of each of four possible journeys (north to west, south to west, north to east, and south to east). In the SW task, rats completed east to south, west to south, east to north, and west to north journeys using the same standard protocol. c, SW performance. Each color-coded circle represents the score of an individual rat. Boxes represent $95 \%$ confidence intervals. Horizontal lines are the mean. Lines connect scores of individuals between sessions. The SW task was learned to criterion in 11.5 trials $(\mathrm{STD} 1=10$ trials, $p=0.3$; mean score: $\mathrm{STD} 1=87 \%, \mathrm{SW} 1=83 \%, p=0.2) . \boldsymbol{d}$, During ALT1, mean performance decreased significantly, but recovered the next day (trials to criterion: $\mathrm{ALT1}=41$, $\mathrm{STD1}=10, p=0.005 ;$ mean score: $\mathrm{STD} 1=85 \%, \mathrm{ALT1}=65 \%, p=0.02 ; \mathrm{STD2}=88 \%, \mathrm{ALT} 2=80 \%, p=0.2)$. $\boldsymbol{e}$, The representative coronal section shows a simultaneous placement of tetrodes in CA1 and CA3. $f$, Cluster-waveform maps of CA1 cells recorded in the first and last STD session demonstrate the stability of recording location. The clusters surrounded by an ellipse contain the waveforms shown in the bottom right. $\boldsymbol{g}$, Recording stability was further determined by calculating the correlation coefficient between the average waveforms recorded during the first and last recording sessions (see Materials and Methods). The graphs show the distribution of waveform correlation coefficients between all recorded cells, in CA1 and CA3, respectively. Waveforms were highly correlated (all $r>0.9$ ), demonstrating high stability.

(SW) task, the start and goal locations were interchanged, so the rat had to adopt opposite spatial trajectories in the same environment. In an altered environment (ALT) task, the STD procedures were used, but local and distal cues were different. We recorded the same CA1 and CA3 cell populations in two sessions each day: in one, the rats performed the STD task, and in the other they performed one of the task variants. The experiment thereby tested how prior training transferred to different learning and memory demands, analyzed hippocampal coding during learning and stable memory performance in the same cell populations, and compared the correlation between memory performance and coding dynamics.

\section{Materials and Methods}

\section{Subjects}

Male Long-Evans rats (Charles River Laboratories) weighed $\sim 350 \mathrm{~g}$, were maintained at $85 \%$ of their free-feeding weight, and were housed individually on a $12 \mathrm{~h}$ light/dark cycle. Training and testing were restricted to the dark phase. All experiments and surgical procedures were carried out in accordance with the guidelines of the Mount Sinai Institutional Animal Care and Use Committee and the NIH.

\section{Apparatus}

A recording room $(2.8 \times 3.2 \mathrm{~m})$ contained a gray, wooden plus maze elevated $82 \mathrm{~cm}$ above the floor with arms $66 \mathrm{~cm}$ long and $6.1 \mathrm{~cm}$ wide, and with walls $2.5 \mathrm{~cm}$ high. The distal end of each arm had a recessed food well $(1 \mathrm{~cm}$ diameter) that contained a Froot Loop made unavailable by a wire screen. A wooden block prevented entrance to the unused start arm. A rectangular waiting platform $(30 \mathrm{~cm}$ wide, elevated $100 \mathrm{~cm}$ ) was located in the southeast corner (Fig. 1b). The room contained several visual cues (e.g., posters). In the ALT task, black curtains and new visual cues covered the walls, the maze surface was covered with brown plastic, and a new wooden block and waiting platform were used. The new waiting platform was placed at the northeast corner. The location and orientation of the maze in the room was not changed.

\section{Recording apparatus}

Twelve independently movable tetrodes made of four twisted $12.7-\mu \mathrm{m}$ diameter nickel-chromium wires (RO-800, Kanthal Precision Technology) 
a
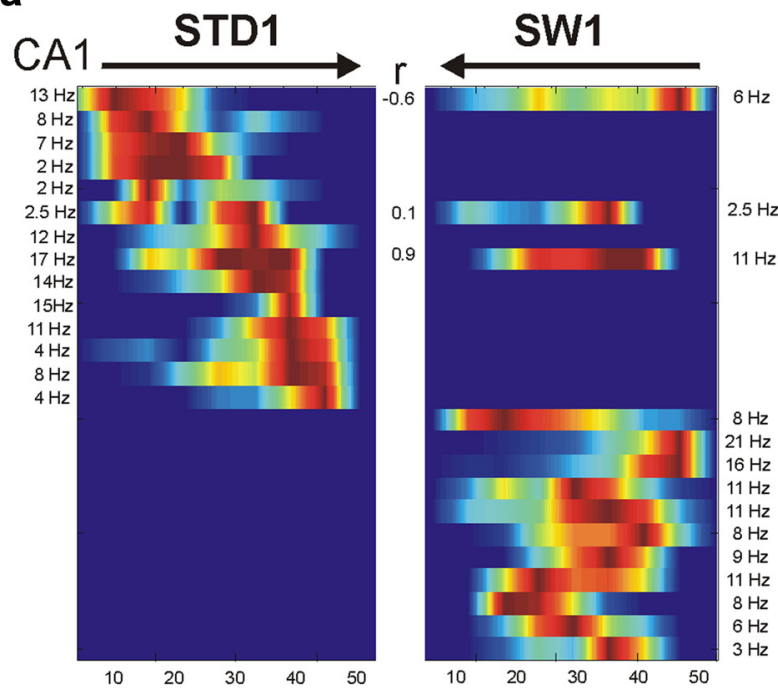

b
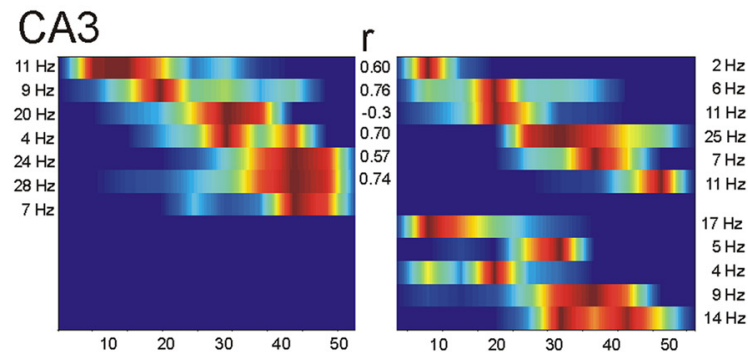

C
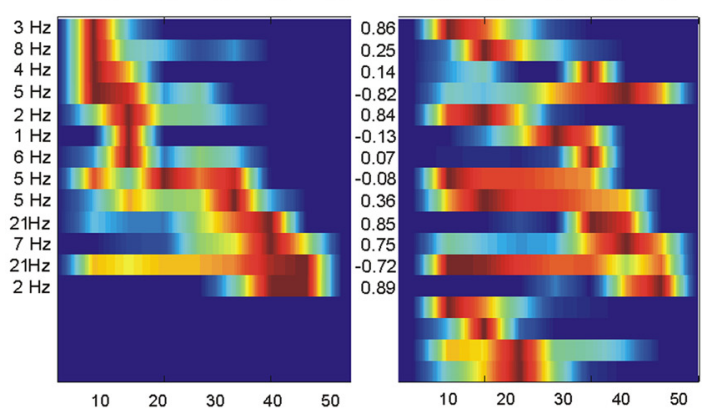

d
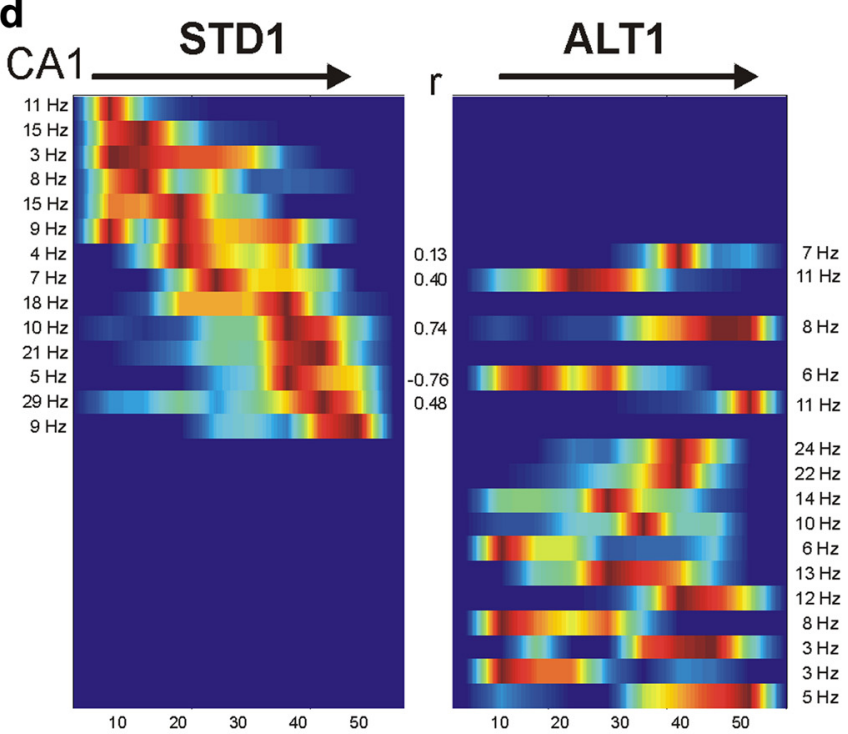

e

\section{CA3}

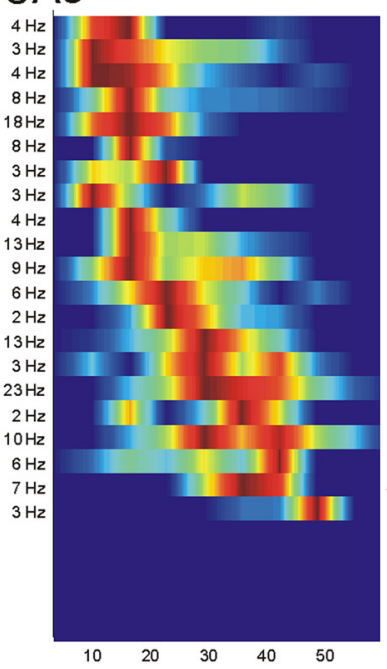

$\begin{array}{lllll}0 & 20 & 30 & 40 & 50\end{array}$ r

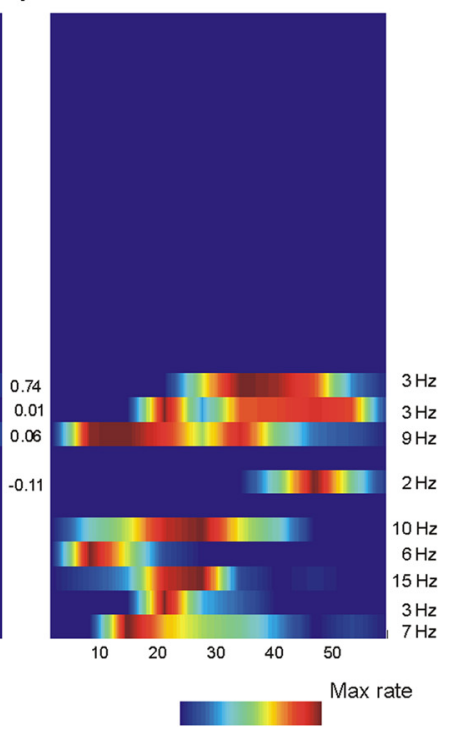

Relative distance from start location on one arm $(\mathrm{cm})$

Normalized firing rate

Figure 2. Firing-rate maps of CA1 and CA3 cells during STD, SW, and ALT tasks. Each panel shows the firing rates of simultaneously recorded neurons. The colored horizontal bars within a panel show the firing-rate distribution of an individual cell on one arm, sorted by the maximal firing rate during the STD. Parallel bars in adjacent panels show the activity of the same cells on the same arm in different tasks. Rate maps were smoothed for this figure only, not in statistical analyses. The color code depicts firing rate normalized to each cell's maximal firing rate. Blue areas on the maps represent $0 \mathrm{~Hz}$. The maximal firing rate of each cell is listed at the edge of each panel; Pearson's $r$ is shown in the center. Arrows on top indicate movement direction. $\boldsymbol{a}-\boldsymbol{c}$, The SW task. Firing-rate maps of CA1 ( $\boldsymbol{a})$ and $C A 3(\boldsymbol{b}, \boldsymbol{c})$ during STD1 (left panels) and SW1 sessions (right panels). $\boldsymbol{a}$ and $\boldsymbol{b}$ show simultaneous recordings in $C A 1$ and $C A 3$, respectively, from the same rat. $\boldsymbol{c}$, CA3 cells recorded from a different rat during the SW session. Note the large CA3 subpopulation that remained active on the same arm between STD and SW. $\boldsymbol{d}, \boldsymbol{e}$, The ALT task. Firing-rate maps of active CA1 (d) and CA3 (e) cells during the STD1 (left) and ALT1 sessions (right). Most CA1 cells were active in a given arm in only one of the tasks. Few CA3 cells were active during the ALT task.

were mounted in a Neuro-hyper-drive assembly (Kopf Instruments). Assemblies were connected to a headstage with source-follower amplifiers (Kopf 54, Neuralynx). Ten color LEDs on the headstage were detected by an overhead color LCD camera to track position and heading $(640 \times 480$ pixel resolution at $60 \mathrm{~Hz}$ ). Neural signals were amplified $2-5 \mathrm{~K}$, filtered between 0.6 and $6 \mathrm{kHz}$, digitized at $32 \mathrm{kHz}$, and stored together with tracking information on a computer (DigitalLynx4S, Neuralynx).

Behavioral training and testing: standard task

Rats were handled (15-30 min/d) for $3 \mathrm{~d}$ and then allowed to explore the maze for $2 \mathrm{~d}$. For the STD task (Fig. 1b), the North and the South arms were used as start arms; the West and the East arms were goal arms.
Before each trial, one-quarter of a Froot Loop was placed in the well at the end of one goal arm. In each trial, the rat was placed on the end of one of the start arms facing the center and had to enter the correct goal arm to find the food. Entering the unrewarded arm for one or more body lengths was recorded as an error, but rats were allowed to correct their choices. The start arm was selected pseudorandomly, so that the same arm was not repeated more than three times consecutively. Between trials, the rat was placed on the waiting platform for 15-20 s. After achieving 9 of 10 correct trials, the goal was reversed and a new block of trials began. Each session was composed of four blocks (Ferbinteanu and Shapiro, 2003). 
a

CA1

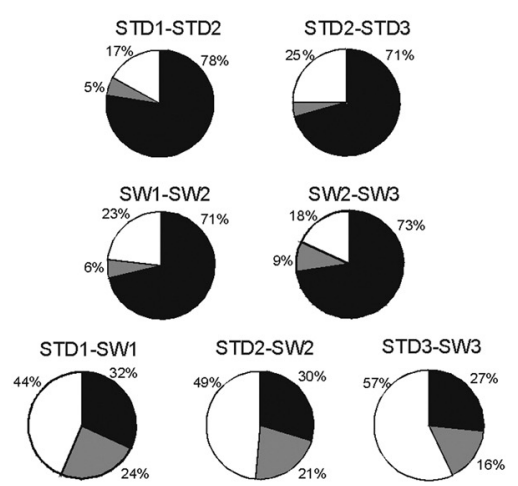

b

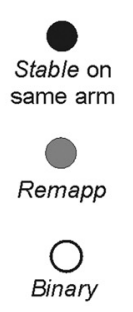

b $\quad$ CA3
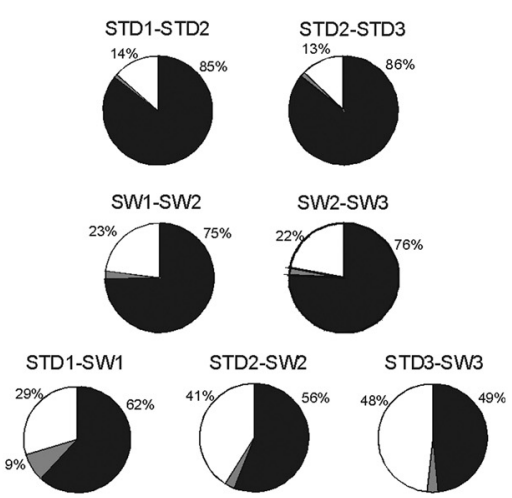

Figure 3. Place-field stability on the same arm between STD and SW sessions. $\boldsymbol{a}, \boldsymbol{b}$, Categorical analyses of CA1 ( $\boldsymbol{a}$ ) and CA3 ( $\boldsymbol{b}$ ) place fields. The pie charts show the distributions of response categories between session pairs (e.g., the STD1-STD2 pie chart in the first row compares between the first and second STD sessions). Response categories were as follows: Stable, cells that maintained a place field on the same arm between sessions; Remap, cells that had a place field in both sessions, but on different arms; Binary, cells that had a place field in only one of the compared sessions. Stable responses were common in both CA1 and CA3 in repeated STD and repeated SW sessions ( $>70 \%$ ). Between STD and SW sessions (bottom row), place fields were significantly more stable on the same arm in CA3 compared with CA1. The levels of Stable response category in CA3 decreased between the first and last recording day $\left(62-49 \% ; \chi^{2}{ }_{(1)}=5, p=0.027\right)$.

The SW task was introduced for the first time during recording sessions using procedures that were identical to the STD, but the East and West arms were used as start arms, and the North and South arms were used as goal arms. The ALT task was also introduced for the first time during recording sessions. New local and distal cues altered the room as described above. All other procedures were identical to the STD task. Four rats performed the SW task, and 6-7 d later, the ALT task. Two rats performed the ALT task first and the SW task later. The task order did not affect firing patterns.

\section{Surgery and stable tetrode placement}

Rats were anesthetized with isoflurane and placed in a stereotaxic frame. The tetrode assembly was fixed to the skull with dental cement, and the tetrodes implanted above the left dorsal hippocampus (AP $3.8 \mathrm{~mm}$, ML $3 \mathrm{~mm}$ from bregma). Two ground wires were attached to skull screws. After the surgery, tetrodes and two reference wires were lowered $1 \mathrm{~mm}$ into the cortex, and rats were injected with Banamine (flunixin meglumine, $1.5 \mathrm{mg} / \mathrm{kg}$, i.m.). When simultaneously recording from CA1 and CA3, six of the tetrodes located laterally were lowered to CA3; the rest were lowered to CA1. Reference wires were lowered to the corpus callosum. Rats were allowed to recover for 6-8 d before behavior testing resumed.

\section{Recording: unit isolation and screening}

Single units with $\geq 3: 1$ signal-to-noise ratios and peaks of $>100 \mu \mathrm{V}$ were discriminated off-line. Complex spike waveforms were characterized by $8-32$ parameters (e.g., peak amplitudes). A custom computer program displayed the represented waveform parameter values as points in a multidimensional space. Points were assigned to distinct clusters using a semiautomated, nonlinear, cluster-cutting algorithm (Ferbinteanu and Shapiro, 2003). The discrimination was further quantified by Mahalanobis distance between clusters in a normalized parameter space. Any pair of clusters $<2$ SDs apart were flagged, and only units with minimal overlap with neighboring clusters and noise were included in subsequent analyses. Putative interneurons (i.e., fast-spiking, short-duration waveforms) were not analyzed.

\section{Data analysis}

Maze behavior. Video tracking data were used to quantify behavior on the maze. The instantaneous location of the rat's head, signaled by LEDs on the headstage, was time stamped and stored in computer files for each session. Post hoc analyses assigned camera pixels to grid units $(6 \times 6 \mathrm{~cm})$. Recordings were separated into four journeys (north to west, north to east, south to west, and south to east during STD and ALT sessions, and west to north, west to south, east to north, and east to south during SW

sessions). Only data recorded in areas restricted to maze arms were analyzed. These areas covered $70-90 \%$ of each arm, where the movement of the rat was reliable. The number of visits, dwell time, heading direction, and running speed were calculated for each grid unit, and the reliability of behavior across different trials was quantified using paired $t$ tests and Pearson's $r$ value. A significant difference $(p<0.05)$ in any of these parameters (except heading direction when comparing STD and SW sessions) excluded the data from further analyses. All analyses excluded activity in the central area of the maze and the waiting platform, where behavior was likely to be different across trials.

Place fields. The firing rate in each grid unit was calculated by dividing the number of spikes by the time spent in each (filtered at a speed of $>2 \mathrm{~cm} / \mathrm{s}$ ). Only cells with a mean firing rate $\geq 1$ spike/s, and only those grid units occupied for $\geq 300 \mathrm{~ms}$ and visited four or more times in each session were analyzed for placefield activity. A place field was defined as an area of two or more adjacent grid units with a mean firing rate of $\geq 1$ spike/s, with three or more spikes per subfield visit and with a firing probability of $>30 \%$ across trials. Categorical analysis of place fields across session types used $\chi^{2}$ statistics.

Firing pattern comparisons. Firing patterns were compared between sessions (e.g., the first STD and SW) for each arm in correct trials only (unless noted otherwise), and included only the restricted area visited in both sessions. Each cell that had a place field on the maze in at least one session was represented by a firing-rate map, an array of grid units corresponding to a maze.

Spatial correlations. Pearson's $r$ quantified the spatial distribution of firing rates of individual cells that had a place field in at least one of the compared sessions. The $r$ values were transformed using Fisher's $z$ transformation and were compared using ANOVA and Tukey's post hoc tests. A firing-rate index compared firing-rate changes in each unit across conditions. The index was computed for each cell in each maze arm by using the following formula to compare grid units: $|(\mathrm{frA}-\mathrm{frB})| /(\mathrm{frA}+$ $\mathrm{frB})$, where frA and $\mathrm{frB}$ were the mean firing rate in conditions $\mathrm{A}$ and $B$, respectively. A firing-rate index $=1$ indicated the maximal rate difference. To determine whether the distributions of firing rates were independent between sessions, each was compared with a randomly generated distribution of indexes (Monte Carlo simulation). The simulation randomly exchanged the firing rates of cells in the SW or ALT sessions, calculated a surrogate firing-rate index, and repeated the procedure 500 times for each rat. Distributions were compared using KolmogorovSmirnov tests. Regression analyses compared the firing rates of the same recorded neurons in each maze arm across all pairs of STD, SW, and ALT sessions. The regressions calculated how well the firing rate of each neuron in a given arm in one session predicted the firing rate of the same neuron in the same arm in another session. For example, the list of mean firing rates for each neuron assessed in the North arm during STD1 was used to predict the firing of the same neurons in the North arm during the STD2. The correlation values and regression coefficients (slopes) assessed each cell's firing-rate stability and dynamics within and between tasks and cell types. Population vectors were constructed by defining 3D arrays (grid unit location $x, y$, mean firing rate of cell $n$ ). Thus, each grid unit was associated with a vector of mean firing rates of the cells that had a place field on that arm in at least one of the sessions to be compared (Leutgeb et al., 2005; Kennedy and Shapiro, 2009). Population vectors were constructed on an arm-by-arm basis and separately for each rat, and distributions of Fisher's $z$ transformation were compared using Kolmogorov-Smirnov tests (see Fig. 9) (ALT 1-ALT 2).

Error trials were analyzed by comparing the firing-rate maps constructed from blocks of four to five trials, on a journey-by-journey basis. 
Error trials were compared with correct trials only if the movement of the rat was statistically indistinguishable between the conditions.

Stability of recording across days. To maximize recording stability, tetrodes were advanced at a rate of $20-30 \mu \mathrm{m} / \mathrm{d}$ after reaching $\sim 200 \mu \mathrm{m}$ above the layer (based on the amplitude of sharp waves and ripples in the slow-wave signal (Buzsáki, 1986). Recording sessions began after tetrodes settled for at least $72 \mathrm{~h}$. The tetrodes were not moved during recording. Electrode stability was assessed off-line by comparing waveforms and cluster distributions qualitatively and quantitatively. Units identified in one session were compared with all other sessions visually and matched based on waveform similarity, cluster location, size, and boundaries (Fig. $1 f$ ). Mahalanobis distances were computed between the clusters. A distance of $>2$ SDs confirmed cluster separation, whereas a distance of $<1$ SD suggested overlap, although all cases were visually inspected to ensure separation and consistency across different sessions. Peak and trough amplitudes ( $1 \mathrm{~ms}, 32$ measures) were extracted from the averaged waveforms and compared by Pearson's $r$ (Kennedy and Shapiro, 2009). The $r$ values were $\geq 0.90$ between the first and last recording sessions (Fig. $1 g$ ). Data acquired from tetrodes that showed altered waveforms between recording sessions were excluded from further analysis.

To further test neuronal stability, we compared waveforms and place fields recorded from the same cell populations in a separate random foraging task (Muller et al., 1987) before and after each experiment. An open square box made of opaque Plexiglas $(80 \mathrm{~cm} / \mathrm{side}, 39 \mathrm{~cm}$ high) with different visual cues on each wall was elevated $85 \mathrm{~cm}$ from the floor and placed in the center of the testing room. Unit recording began after the rats were familiar with the box and searched readily for chocolate sprinkles. We recorded unit activity in the random foraging task in two $10 \mathrm{~min}$ sessions, once before the first STD recording session on Day 1, and again a few minutes after the last SW or ALT sessions on Day 3. More than $76 \%$ of CA1 and CA3 cells had stable place fields in the box in both random foraging sessions. The mean spatial correlation and the median population vector correlation were $\geq 0.6$ between the first and last session in both CA1 and CA3, consistent with previous quantitative studies (Kentros et al., 1998; Leutgeb et al., 2004). The results provide converging evidence that the same populations of cells were recorded throughout the 3 recording days.

Histology

Rats were anesthetized with sodium pentobarbital (100 mg/kg, i.p.). A DC current was passed through each tetrode ( $40 \mu \mathrm{A}, 4 \mathrm{~s})$ to induce a lesion. Brain sections $(35 \mu \mathrm{m})$ were labeled with formol-thionin stain.

\section{Results}

\section{New learning was facilitated by prior experience}

Initial training in the STD task required six to seven daily sessions (240-420 trials). Learning was more rapid in the SW and ALT tasks. During the first SW session (SW1), overall performance declined only slightly, rats reached criterion performance almost immediately, and good performance was stable throughout subsequent sessions (Fig. 1c). During the first ALT session (ALT1), overall performance declined significantly and returned to criterion in the second session (Fig. 1d). The ALT task was learned more slowly than the SW task (mean trials to criterion: ALT1 = 41 , SW1 $\left.=11.5 ; t_{(4)}=-5.3, p<0.01\right)$. Thus, adopting new spatial trajectories in a highly familiar environment was significantly faster than learning new spatial discriminations.

To investigate how the hippocampal coding that was established in the STD task changed during SW and ALT learning, we recorded CA1 (363) and CA3 (215) complex-spike units in six rats as they performed STD and either SW or ALT tasks. Some experiments recorded CA1 and CA3 cells simultaneously (2 rats each in STD-SW and STD-ALT comparisons) (Fig. 1e). Together, 136 CA1 (3 rats) and 143 CA3 (5 rats) cells were compared in STD and SW tasks; 227 CA1 (4 rats) and 72 CA3 (3 rats) cells were compared in STD and ALT tasks. Recording stability was assessed by comparing tetrode waveforms across sessions (Fig. 1f,g), and

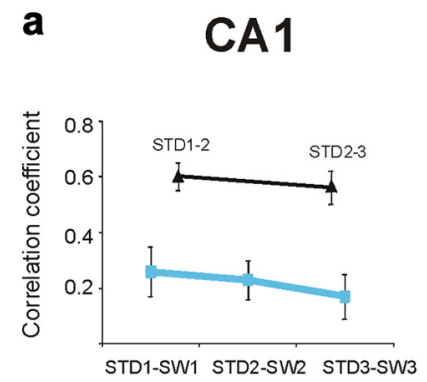

b $\quad$ CA3
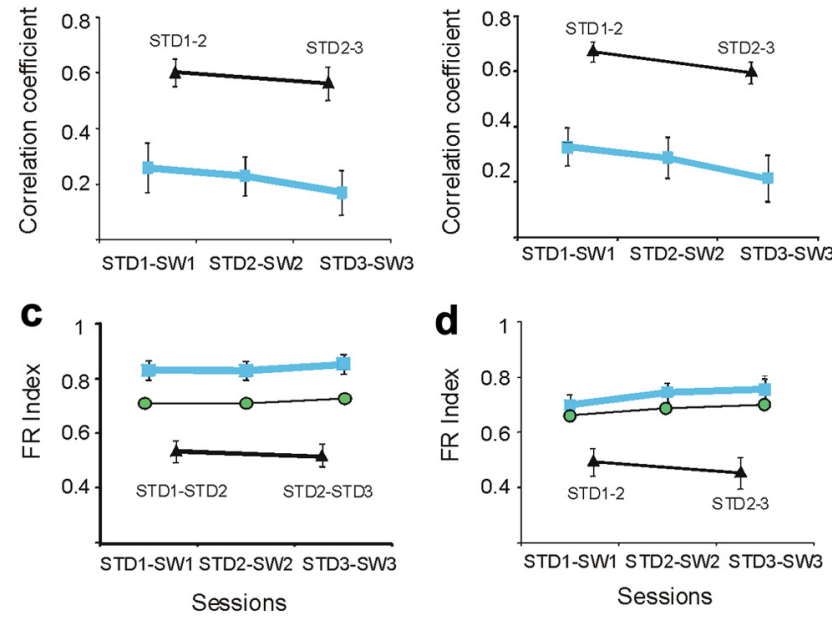

Figure 4. Spatial and rate codes differed between STD and SW in both CA1 and CA3 cells. $\boldsymbol{a}$, $\boldsymbol{b}$, Spatial correlations of individual CA1 ( $\boldsymbol{a}$ ) and CA3 ( $\boldsymbol{b})$ cells between STD and SW sessions were significantly lower than the correlations computed for repeated STD sessions. The blue boxes indicate the mean correlations at different recording days between STD and SW sessions (labeled by the $x$-axes; error bars are SEM). The black triangles indicate the mean correlations between repeated STD sessions. The correlations between repeated SW sessions were not statistically distinguishable from repeated STD sessions (data not shown; $p>0.36$ ). $\boldsymbol{c}, \boldsymbol{d}$, Firingrate changes across sessions in individual cells were quantified by a firing-rate index (see Materials and Methods). A higher value indicated a higher rate change. $c$, In CA1, the average firing-rate changes were significantly greater between the STD and SW sessions (blue boxes) than between repeated STD sessions (black triangles), and were also greater than the rate changes expected by independent remapping (green circles; mean \pm SEM, STD1-SW1: observed rate index $=0.83 \pm 0.03$, independent rate index $=0.71 \pm 0.01, \mathrm{KS}=0.2, p<0.01$ ) (firing-rate index across rats: $C A 1=0.78,0.75,0.9)$. The average firing-rate indices expected by independent remapping were calculated after permuting the firing rates of individual cells in the SW session and calculating simulated firing-rate indices, see Materials and Methods (Leutgeb et al., 2004). $\boldsymbol{d}$, In contrast to CA1, the average CA3 firing-rate changes between STD and SW were not different from the average rate changes expected by independent remapping (mean \pm SEM, STD1-SW1: observed mean $=0.7 \pm 0.04$, simulated mean $=0.66 \pm 0.01$, $\mathrm{KS}=0.07, p=0.8$ ) (firing-rate index across rats: $\mathrm{CA} 3=0.57,0.6,0.7,0.68,0.7$ ).

by comparing place-field stability in recordings made before and after the experimental sessions as rats performed a random foraging task (see Materials and Methods). Only units with stable waveforms were included in subsequent analyses.

\section{Most CA3 cells maintained place fields in the same maze arms in the STD and SW tasks}

The rats acquired the SW almost immediately, showing that some information transferred between the STD and SW tasks. If the hippocampus contributed to this transfer, then some aspect of hippocampal coding in the STD task might persist in the SW task. Because the maze arms occupied the same locations in the room, neurons that fired in the same arms could provide a representational "bridge" between the STD and SW sessions. To test this possibility, we identified place fields using standard methods (Ferbinteanu and Shapiro, 2003), and categorized units as stable, remapped, or binary. A stable unit had a place field on the same arm in both STD and SW tasks (Fig. $2 a$, top bar). A remapped unit had a place field that occupied different arms in the STD and SW tasks. A binary unit had a place field in either the STD or the SW task, but not both. Stable responses were more common in CA3 $(62 \%)$ than would be expected by chance $(25 \%)$, and were significantly more common than stable responses in CA1 $(32 \%)$ $\left(\chi_{(2)}^{2}=22, p<0.001\right)$ (Fig. 3). Hence, many CA3 cells indicated 
a

CA1
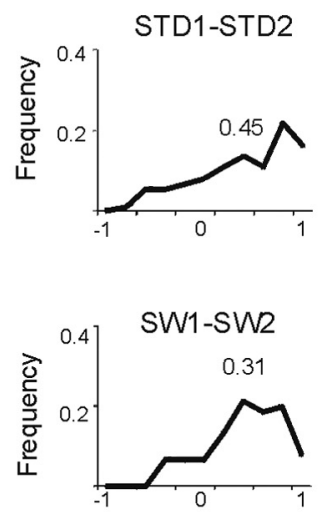

b

CA3
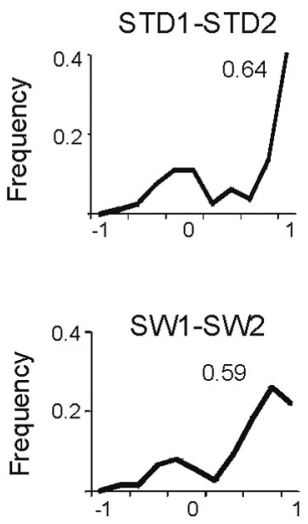

STD2-STD3
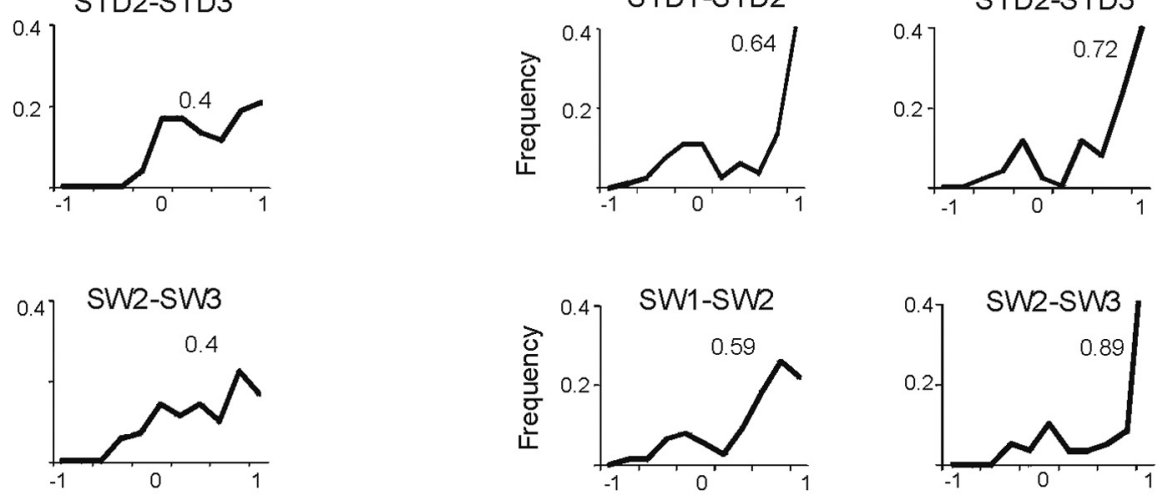
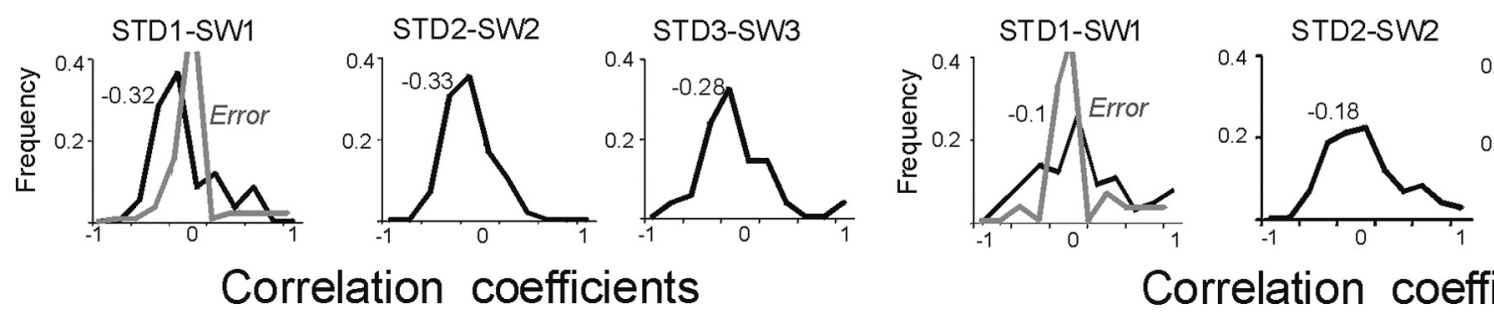

Figure 5. CA1 population vectors were anticorrelated between STD and SW, whereas $C A 3$ vectors were uncorrelated. $\boldsymbol{a}, \boldsymbol{b}$, Population vector analyses for $C A 1$ (a) and $C A 3(\boldsymbol{b})$. The plots show the frequencies of population vector correlations between different sessions. Top row, between repeated STD sessions; middle row, between repeated SW sessions; lower row, between STD and SW sessions. The values above the distributions depict medians. Between STD and SW sessions, CA1 population vectors were more anticorrelated (median $=-0.32$ ), compared with CA3 population vectors (median $=-0.1$ ). Distributions of correlations during error trials (comparing error SW1 to correct STD1 trials) are marked in gray. In CA1, most correlations between error SW and correct STD trials became less anticorrelated (median was 0 ). Unlike CA1, CA3 population vector correlations did not distinguish correct from error trials (median correlations were 0 in correct or error trials).

that the rat was on the same maze arm in the STD and SW tasks, even as heading direction was reversed and goal locations changed. The stable coding may have signaled common spatial information in both the STD and SW sessions, thereby contributing to the rapid learning during the SW task. Future experiments are required to determine whether the proportion of stable responses are a consequence of the initial training or other experimental features.

Because CA3 activity can modulate CA1 neurons via Schaffer collaterals (Witter, 2007), we tested whether the subset of CA1 place fields that remained active in both tasks reflected the spatial stability of CA3 fields. If stable CA3 cells influenced the CA1 cells that had place fields in both tasks, then these CA1 cells should also have fields in the same arms across the STD and SW. Indeed, CA1 cells with place fields in both tasks were significantly more likely to occupy the same arm in the STD and SW tasks than to remap to a different arm (between STD1 and SW1: $\chi_{(1)}^{2}=13.6, p<$ $0.001)$. Moreover, the proportion of stable place fields within this active CA1 subset was similar to the overall proportion of stable CA3 place fields (57\%). Future experiments will have to determine whether these "arm-anchored" CA1 place fields reflected the direct influence of stable CA3 cells or other common inputs.

\section{CA1 and CA3 place fields remapped during switch trials}

The SW task exchanged the start and goal, so rats made opposite trajectories through the maze. The categorical analysis just described showed that most CA3 cells had place fields on the same arm in the STD and SW tasks, despite opposite heading direction. Prior studies using similar behavioral conditions found $60-80 \%$ of CA1 and CA3 cells had place fields that differed significantly when the firing rate along the maze arms was compared (McNaughton et al., 1983; Markus et al., 1995). To quantify the spatial distribution of firing rates along each arm, we compared the STD and SW sessions using Pearson's $r$ (Fig. $4 a, b$ ). Spatial correlations were significantly lower between STD and SW sessions than between repeated STD sessions, in both CA1 and CA3 cells (mean $r \pm$ SEM, in CA1: STD1-STD2 = $0.6 \pm 0.05$; STD1$\mathrm{SW} 1=0.26 \pm 0.09, F_{(6,632)}=10, p<0.001$, Tukey's post hoc test; in CA3: STD1-STD2 $=0.67 \pm 0.06$; STD1-SW1 $=0.34 \pm 0.07$, $F_{(6,330)}=9, p<0.001$; mean $r$ in each rat: $\mathrm{CA} 1=0,0.17$, and 0.12 ; CA3 $=0.2,0.25,0.27,0.12$, and 0.15). Moreover, the effects of task on spatial correlations were similar in CA1 and CA3 (e.g., day 1: $t_{(145)}=1.1 p=0.2$ ). Although the correlation analysis replicated the basic observations in the prior and current experiments, the categorical analysis showed that CA3 was more likely than CA1 to indicate the same maze arm across tasks, and suggested that CA3 cells may provide a "coarse code" that indicated common locations or memory discriminanda.

Overall, the results are consistent with prior observations that the activity of most hippocampal cells is strongly influenced by heading direction when rats traverse linear tracks (McNaughton et al., 1983; Muller and Kubie, 1987; Markus et al., 1995; Gothard et al., 1996). A new observation is that CA3 cells were more likely than CA1 cells to fire in the same arm despite opposite trajectories. This observation could be considered consistent with the idea that rate remapping in CA3 underlies directional place fields as well as other signals that distinguish among episodes that occur in the same place (Leutgeb et al., 2005). From this point of view, CA3 place fields should occupy the same places but have different firing rates in the STD and SW tasks, and the magnitude of firing- 
rate differences should be larger in CA 3 than CA1. To test this prediction, we quantified rate changes in individual cells across sessions using a firing-rate index (Fig. $4 c, d$ ). The index showed significantly greater rate changes between STD and SW sessions than between repeated STD session in both CA1 and CA3 (Fig. $4 c, d)$. The firing-rate changes differed in CA1 and CA3, however. CA1 firing rates changed significantly more than would be expected by independent remapping (Fig. 4c, green circles), whereas CA3 firing rates changed to the same extent as would be expected by chance (Fig. $4 d$ ). The stronger response of CA1 cells to the SW was verified by a regression analysis that compared each cell's firing rate in each arm across pairs of recording sessions. CA1 firing rates were anticorrelated and significantly less than zero, whereas CA3 firing rates were uncorrelated (STD vs SW, CA1: $r=-0.14, \beta=-0.12$, vs $0: t_{(7)}=6.6, p<0.05$; CA3: $r=-0.07, \beta=-0.05$, vs $\left.0: t_{(7)}=1.7, p=0.16\right)$. In other words, the magnitude of the firing-rate change between the STD and SW was higher in CA1 than CA3 (compare to Leutgeb et al., 2005). Although "arm coding" appeared to provide a coarse spatial code that overlapped in the two tasks, and though these "bridging cells" were more common in CA3 than CA1, the present results did not reveal independent rate and global remapping. CA3 place fields were typically maintained in the same maze arm across the STD the SW, but the spatial correlations were low, and the firingrate changes were greater in CA1 than CA3.

\section{Anticorrelated CA1 population vectors were associated with switch performance}

Population vector correlations compared the firing rates of simultaneously recorded cells across tasks and sessions (Fig. 5) (Leutgeb et al., 2005; Kennedy and Shapiro, 2009). Population vectors were stable across repeated STD sessions (median $r$, STD1-STD2: CA1 $=0.45$, CA3 $=0.64)$. As expected by the spatial correlations and rate changes described above, population vectors strongly distinguished between STD and SW sessions. The population vector analysis revealed further that CA1 codes in the STD and SW tasks were anticorrelated (median $r$, STD1-SW1: $\mathrm{CA} 1=-0.3$, CA3 $=-0.1$; CA1 vs CA3: KS $>0.25, p<0.05$ ). The anticorrelation in CA1 was consistent across rats and recording days, and occurred because most of the cells active on one arm during one task were entirely silent in that arm in the other task (Fig. 2a), consistent with the firing-rate index results above (population vectors across rats: $\mathrm{CA} 1=-0.4,-0.37,-0.3$; CA3 $=$ $-0.15,-0.1,0,-0.2)$. The anticorrelation was not caused by reversed firing sequences, as it was maintained when the SW rate maps were virtually inverted during off-line analysis (median $r$, STD1 vs inverted-SW1 $=-0.33$ ). Anticorrelation was also not a result of place fields accumulating near the new goal locations (Hollup et al., 2001; Fyhn et al., 2002), because the observed place-fields remapping into the different maze arms was not different than the remapping expected by chance $\left(\chi_{(2)}^{2}=1, p=0.3\right)$.

Although directional coding could provide a simple explanation for the remapping observed between STD and SW sessions, several factors mitigate against it. First, remapping differed in CA3 and CA1 neurons, demonstrating partial spatial stability in CA3, and anticorrelation in CA1. Second, other studies show that CA1 firing dynamics correlated with memory discrimination performance even as heading direction and other behavioral variables were kept constant (Ferbinteanu and Shapiro, 2003; Kennedy and Shapiro, 2009). If the anticorrelation of CA1 population vectors was related exclusively to directional coding, then anticorrelation should occur each time the rat made a reversed trajectory, regardless of task performance. We found, however, a

$\mathbf{a}$
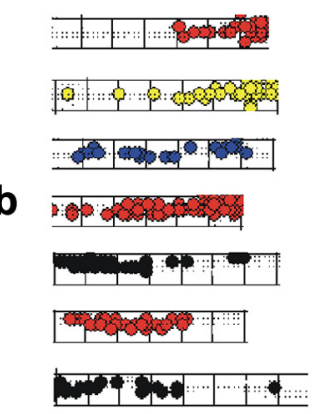

c Correct SW

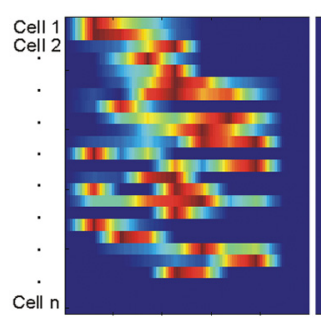

Correct SW
Error SW

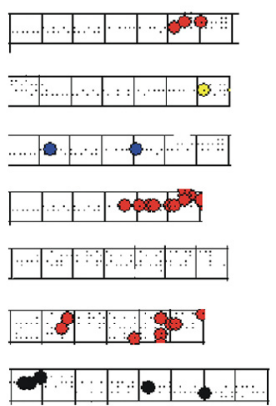

Error SW

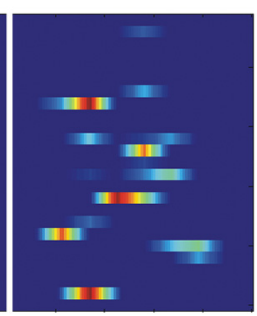

Figure 6. Firing patterns across correct and error trials. During errors SW trials many CA1 and CA3 cells had lower firing rates compared with correct SW or STD trials (mean rate on an arm, $\mathrm{Hz} \pm \mathrm{SEM}$, correct trials: $\mathrm{STD} 1=2.7 \pm 0.2, \mathrm{SW} 1=3 \pm 0.2$; errors $=0.9 \pm 0.2, \mathrm{~F}_{(2,1374)}=$ $25, p<0.0001$, Tukey's post-test). Error trials were included in the analysis only if overt behavior (speed and direction) was consistent. The average speed was $4.5 \%$ lower in error trials compared with correct trials, and different speeds on the entire arms or within the firing fields were poorly correlated with different rates $\left(r^{2}=0.024 ; r^{2}=0.01\right) . \boldsymbol{a}, \boldsymbol{b}$, Representative examples of spiking activity of CA1 (a) and CA3 (b) cells on different arms of the maze. The squares show grid units, gray dots show visits, and colored dots represent spikes. Four correct and four error trials in the SW task are shown. c, Color-coded firing-rate map showing population activity of $C A 1$ cells during correct $S W$ trials and error $S W$ trials (activity was averaged across four trials). Firing rates were normalized for each cell across correct and error trials. The average firing rate of $C A 1$ and $C A 3$ cells did not decrease during errors in the ALT task (mean rate $\pm S E M$, ALT: correct $=2.87 \pm 0.2$, error $=2.9 \pm 0.3, t_{(524)}=-0.09, p=0.92$ ).

that the anticorrelation of CA1 population vectors covaried with SW performance $(r=-0.46, p<0.0001)$ and diminished when rats made errors (median $r$ between STD1 and SW1: errors $=0$; correct vs error trials: $t_{(202)}=-3.1, p=0.002$ ) (Fig. 5a). In contrast, CA3 population vectors were uncorrelated in both correct and error trials (median $r$ between SW 1 and STD1: correct vs error trials $=0, t_{(170)}=0.28, p=0.7$ ) (Fig. $5 b$ ). Moreover, firing rates declined in both CA1 and CA3 during error trials even as running speed was largely unchanged (Fig. 6). Thus, anticorrelated CA1 codes predicted performance in the SW task and did not merely reflect directional place fields.

Firing patterns distinguished between the STD and ALT tasks, and CA3 place fields were absent

Most distal room and local maze cues were changed in the ALT task, and memory performance was initially impaired (Fig. 1d). As would be expected in two distinct environments, $60 \%$ of CA1 and $80 \%$ of CA3 place fields were categorized as binary between STD1 and ALT1 sessions (i.e., were active in one session only), consistent with global remapping (Fig. 7). Across STD and ALT sessions, spatial correlations of individual place fields were significantly lower, and firing-rate changes were significantly greater than between repeated STD sessions (Fig. 8). Population vectors were more anticorrelated in CA1 than CA3 in the first ALT session, as was observed during the SW task $(\mathrm{KS}=0.35, p<0.05)$, but $\mathrm{CA} 3$ population vectors became more anticorrelated in later 
a CA1
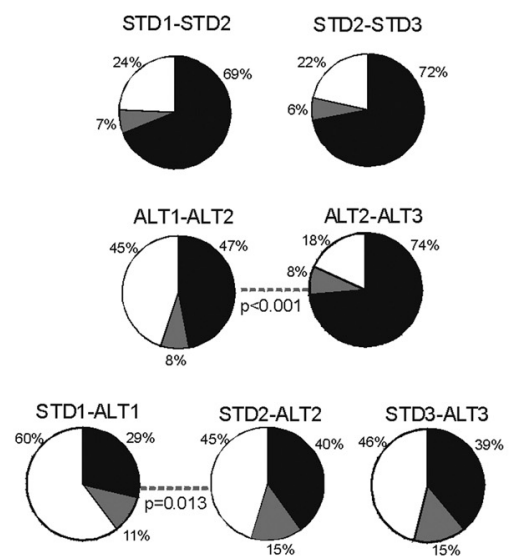

b

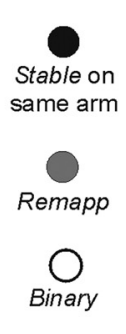

b
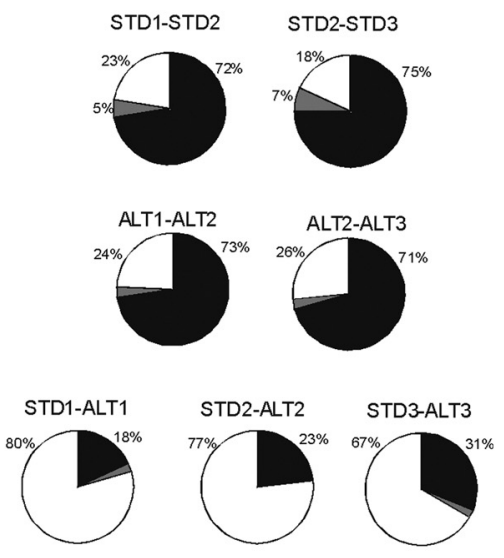

Figure 7. Categorical analysis of hippocampal place fields in STD and ALT sessions. $\boldsymbol{a}, \boldsymbol{b}$, The pie charts show distribution of response categories between session pairs in CA1 $(\boldsymbol{a})$, and $\mathrm{CA} 3(\boldsymbol{b})$ cells. Dotted lines indicate a statistically significant difference between a session pair. Stable responses were common in both CA1 and CA3 between repeated STD sessions. Stable responses were also common in CA3 cells between repeated ALT sessions, regardless of improved memory performance across these sessions. Two significant changes were observed between the first and second ALT sessions in CA1 fields: $47 \%$ were stable between the first and second ALT sessions, and the stability increased significantly in later sessions (see increased Stable category during ALT2-ALT3, second row). Twenty-nine percent of CA1 fields were stable between STD1 and ALT1 sessions, and the stability increased significantly in later STD and ALT comparisons (bottom row). In CA3, most cells had a place field in either STD or ALT, but not both ( $\boldsymbol{b}$, bottom row). a

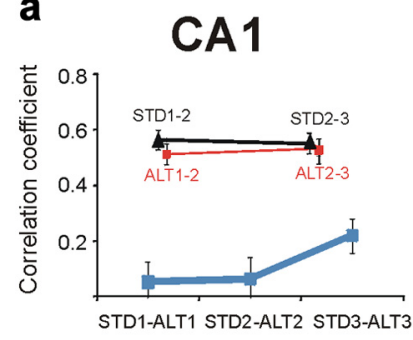

C

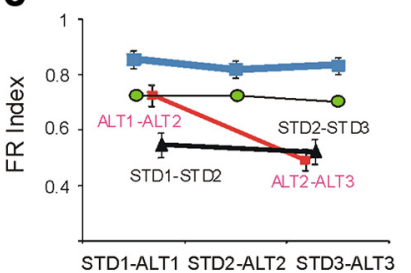

b

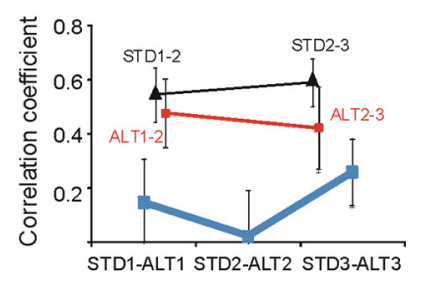

d

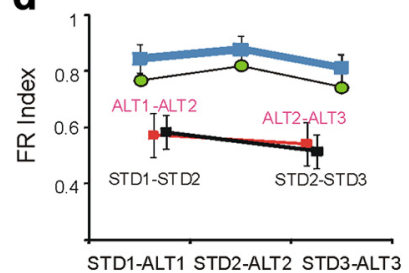

Figure 8. Spatial and rate codes differed between STD and ALT in both CA1 and CA3 cells. $\boldsymbol{a}$, $\boldsymbol{b}$, Spatial correlations of individual CA1 (a) and CA3 (b) cells between STD and ALT sessions were significantly lower than the correlations computed for repeated STD sessions. The blue boxes indicate the mean correlations at different recording days between STD and ALT sessions (labeled by the $x$-axes; error bars are SEM). Black triangles and red boxes indicate the mean correlations between repeated STD and ALT sessions, respectively. (mean Pearson's $r$ between STD1 and ALT1 in individual rats: $C A 1=0,0,0.1,0.01 ; C A 3=0,0.14,0.1) \boldsymbol{c}, \boldsymbol{d}$, Firing-rate changes across sessions in individual cells were quantified by a firing-rate index (see Materials and Methods). $c$, In CA1, the average firing-rate changes were significantly greater between the STD and ALT sessions than between repeated STD sessions, and were also greater than the rate changes expected by independent remapping (green circles; mean \pm SEM, STD1-ALT1: observed firing-rate index $=0.86 \pm 0.02$, independent index: $\mathrm{CA} 1=0.73 \pm 0.01, \mathrm{KS}=0.21$, $p<0.01$ ) (mean firing-rate index between STD1 and ALT1 across individual rats: $C A 1=0.85$, $0.86,0.84,0.9) ; C A 3=0.72,0.9,0.84)$. Firing rates of $\mathrm{CA} 1$ cells were unstable between ALT1 and ALT2 sessions, as indicated by the high firing-rate index calculated between the ALT1 and ALT2 sessions. $\boldsymbol{d}$, In contrast to CA1, CA3 firing-rate changes between STD and ALT were not different from the rate changes expected by independent remapping (mean \pm SEM, STD1-ALT1: observed mean $=0.85 \pm 0.05$, independent index: $\mathrm{CA} 3=0.77 \pm$ $0.01, \mathrm{KS}=0.14, p=0.8)$. sessions (Fig. 9). Overall, CA1 population vectors were consistently anticorrelated in both the ALT and SW when compared with the STD, suggesting that the hippocampus reflected an active process that distinguished the tasks. In contrast to the SW task, however, memory performance during the ALT task was not predicted by CA1 anticorrelation (Fig. 9a, gray line).

The most striking observation in the ALT task, however, was that most CA3 cells had no place field on the maze throughout the three ALT sessions, even as the proportion of CA1 neurons with place fields was maintained (ratio of place field/cells in CA3 in each rat: STD1-3: 0.44, 0.43, 0.42; ALT1-3: 0.15, 0.18, 0.18; STD1-ALT1: $Z=3.6, p=0.001$; STD3ALT3: $Z=3.2, p=0.003$, Fisher's exact test) (Figs. 2e, 10). The CA3 cells that were silent on the maze fired occasionally on the waiting platform between trials. Moreover, the same cells maintained activity and place fields in the STD sessions recorded on the same, prior, and following days, and in the random foraging task at the start and end of the experiment. The few CA3 cells that had place fields in the ALT task fired at normal rates (mean infield rate: STD1 and ALT1 $\approx 5 \mathrm{~Hz}$ ), but in different patterns than in the STD. A regression analysis compared each cell's firing rate in each arm across pairs of recording sessions and found that CA3 firing rates were anticorrelated and significantly less than zero, whereas CA1 firing rates were uncorrelated (STD vs ALT, CA3: $r=-0.10, \beta=-0.19$, vs $0: t_{(7)}=4.6, p<0.05$; CA1: $r=-0.05, \beta=-0.04$, vs $\left.0: t_{(7)}=1.9, p=0.08\right)$. The consistent number of CA1 place fields in the ALT and STD tasks (Fig. 10a) demonstrate that CA1 activity was maintained despite reduced CA3 input.

\section{CA1 place fields stability increased with ALT performance}

Though the proportion of CA1 cells with place fields on the maze was maintained in the ALT task, the place fields changed across sessions. When rats first explore an unfamiliar environment, CA1 unit activity is initially unstable and diffuse, but stable place fields form within 5-30 min (Wilson and McNaughton, 1993; Karlsson and Frank, 2008) and can persist for months (Thompson and Best, 1990). Consistent with these findings, CA1 activity was unstable between ALT1 and ALT2, and stable between ALT2 and ALT3, as shown by spatial correlations, population vectors, and firing-rate changes (Figs. $7 a, 8 c, 9 a$ ). The rapid formation of new, stable CA1 place fields correlated with memory performance in the ALT task, so that memory performance during ALT1 was predicted by high population vector correlations between ALT1 and ALT2 $(r=0.26, p=0.015)$ (Fig. 11a). The increased population vector correlation across repeated ALT sessions also correlated with improving performance (Fig. 11b) $(r=0.39, p<$ 0.0001), but not with differences in running speed (Fig. 11c).

As CA1 place fields stabilized across ALT sessions, they also became more similar to those recorded during the STD sessions. Only $29 \%$ of CA1 neurons remained active on the same arm in both the STD1 and ALT1 sessions (Fig. 7a, stable responses). Place fields were stable across the second and third ALT sessions, when memory performance returned to baseline (stable: STD1- 
a

\section{CA1}
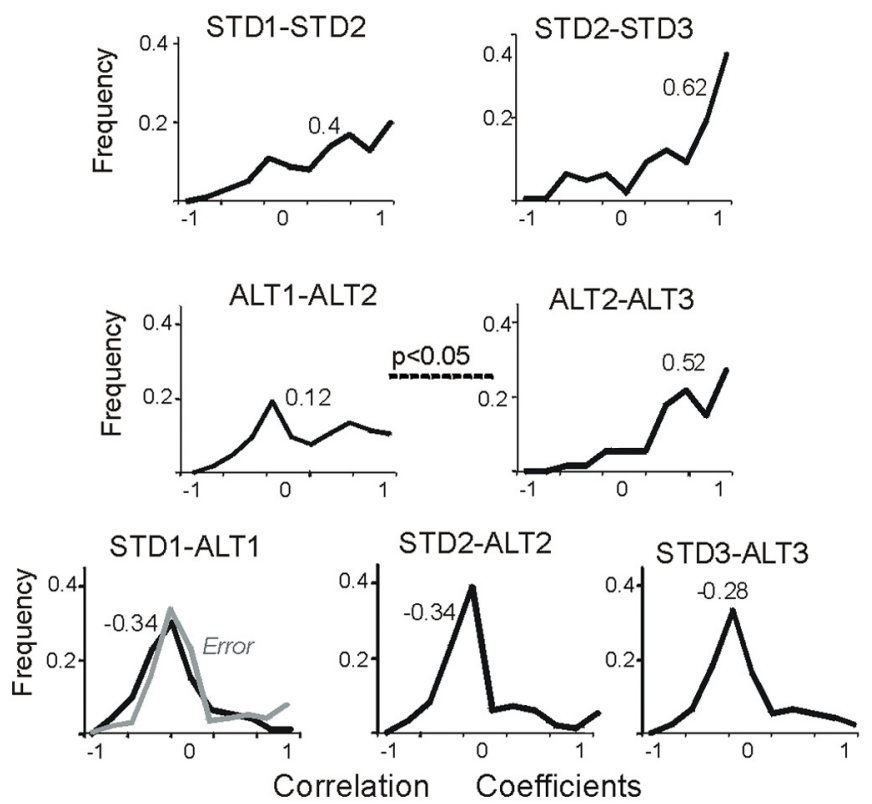

b

\section{CA3}
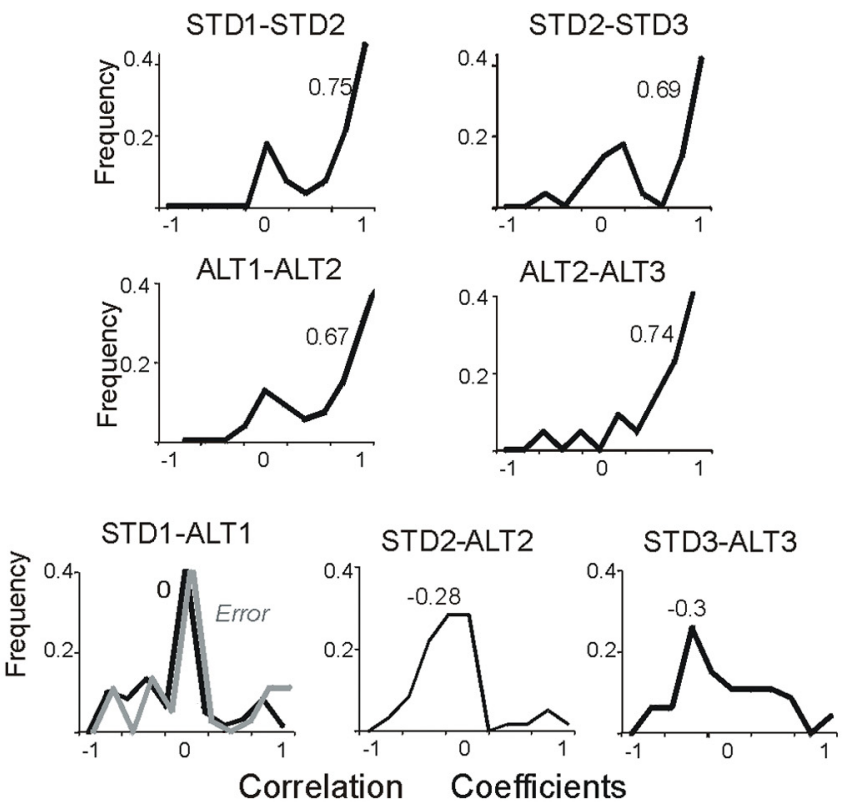

Figure 9. Population vector correlation analysis in STD and ALT sessions. $\boldsymbol{a}, \boldsymbol{b}$, Population vector analyses for CA1 (a) and CA3 ( $\boldsymbol{b})$. The plots show the frequencies of population vector correlations between different sessions. Most CA1 population vectors were anticorrelated between STD and ALT (median $=-0.34$ ), compared with population vectors in CA3 (median $=0$ ) (median population vector correlation between STD1 and ALT1, individual rats: CA1 $=-0.26,-0.29,-0.35,-0.5 ; C A 3=0,0,-0.13)$. In later sessions, many CA3 population vectors became anticorrelated as well (median $r$ in STD2-ALT2: CA1 $=-0.34, C A 3=-0.28, \mathrm{KS}=0.1, p>0.05$ ). Distributions of error trials in ALT1 are shown by gray lines. In CA1, the correlations between error ALT and correct STD trials remained anticorrelated (median was - 0.21). CA3 population vector correlations did not distinguish correct from error trials. Consistent with the categorical and the firing-rate index analyses (Figs. 7, 8), correlations of population vectors were low between the ALT1 and ALT2 sessions, but increased significantly between the ALT2 and ALT3 sessions.
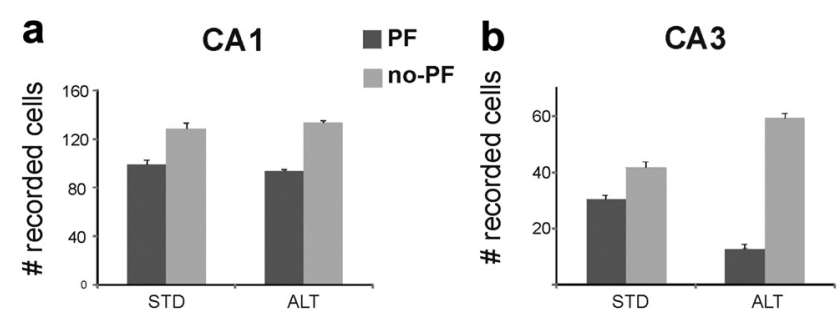

Figure 10. CA3 place fields were absent on the arms during the ALT task. $\boldsymbol{a}, \boldsymbol{b}$, The bar graphs show the average number of identified $\mathrm{CA} 1(\boldsymbol{a})$ and $\mathrm{CA} 3(\boldsymbol{b})$ cells across the 3 recording days for STD and ALT sessions. The number of cells that had a place field (PF) on at least one maze arm is shown by the dark gray bars; the numbers of cells that had no place field in any arm are in light gray. Cells were identified as spike clusters in recordings that included during the intertrial interval on the waiting platform and areas of the maze excluded from the analysis (e.g., during reward consumption). The proportions of CA1 cells with place fields on the arms ( $\sim 40 \%)$ were consistent in all STD and ALT recording sessions and were similar to prior studies. The number of CA3 cells with place fields declined significantly during all ALT sessions (place fields/total recorded cells across rats: $3 / 26,3 / 25,6 / 21$ in ALT1; average ratio across ALT sessions: $0.17,0.18$, 0.18). The cells that had place fields in the STD but not the ALT (e.g., Fig. 2e) sometimes fired on the waiting platform. CA3 place fields tended to be larger during the ALT than the STD sessions, but the difference was not statistically significant (mean place field size, $\mathrm{Cm}^{2} \pm$ SD: STD1 $=$ $118 \pm 65, A L T 1=151 \pm 68$ ). The proportions of $C A 1$ and $C A 3$ cells that had a place field were unchanged between STD and SW (data not shown).

ALT1 $=29 \%$; STD2-ALT2 $=40 \% ; \chi^{2}{ }_{(2)}=8, p=0.013$; increased stable responses: $120-175 \%$ across rats). With repeated experience in the two environments, in other words, CA1 place fields recorded in the STD and the ALT became more similar as performance improved. Increased stability was not a result of place fields clustering around goal locations, which were in the same place in the STD and ALT sessions (Hollup et al., 2001; Fyhn

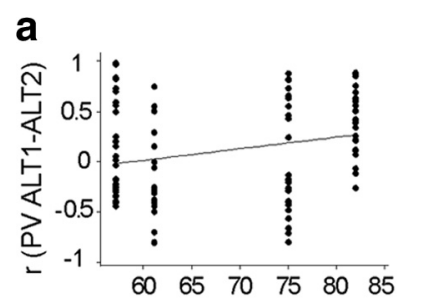

Performance score ALT1 (\%)
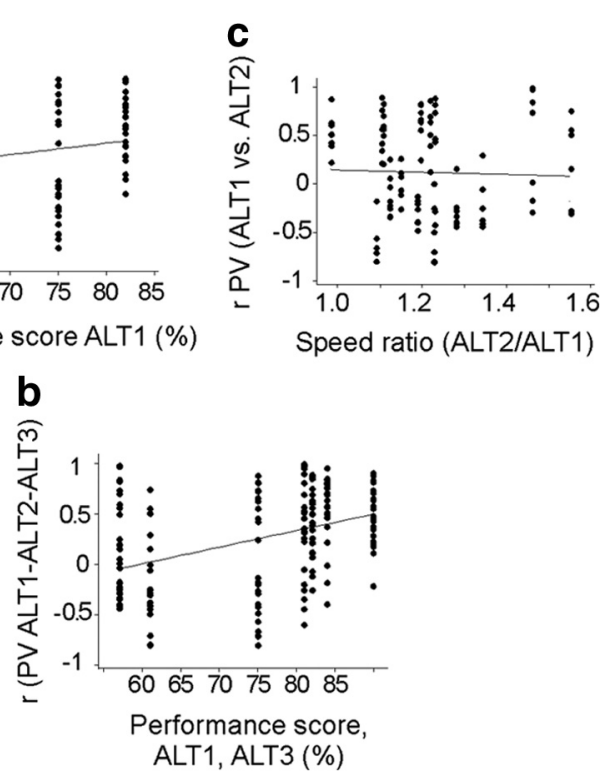

Figure 11. Population vectors in CA1 stabilized as performance improved during repeated ALT sessions. $\boldsymbol{a}$, Performance during ALT1 was predicted by the similarity of population vectors in ALT1 and ALT2 sessions. The correlation $(r=0.26, p=0.015)$ suggests that better performance during the first ALT session was related to stable population vectors that were maintained the next day. Each column of symbols shows memory performance and population vector correlations for one rat. $\boldsymbol{b}$, The association between performance and population coding stability appeared to strengthen in ALT3 sessions, when the population vector correlations among the three ALT sessions were included $(r=0.39, p<0.0001)$. c Differences in running speeds across ALT1 and ALT2 were unrelated to population vector correlations across the ALT sessions $(r=0, p=0.7)$. 
et al., 2002), because new place fields appeared with similar probabilities on start and goal arms $\left(\chi_{(1)}^{2}=1.2, p=0.3\right)$.

In contrast to CA1, the few CA3 place fields recorded in the ALT task were consistently binary (i.e., only active in either the STD or the ALT), and the proportion did not change significantly with performance (Fig. $7 b$ ). Though hippocampal place fields distinguished between the STD and ALT, CA3 and CA1 provided different signals. Subsets of CA1 place fields developed more similar representations over repeated sessions, whereas CA3 place fields strongly and stably discriminated the two tasks. Furthermore, both the stability of CA1 place fields in the ALT task and the similarity of the place fields in the ALT and STD tasks correlated with memory performance, independent of CA3.

\section{Discussion}

Few studies of place-field dynamics in rats exploring unfamiliar environments or taking opposite trajectories include direct measures of learning and memory performance. Here, rats trained to perform a familiar, hippocampus-dependent memory task in new circumstances showed facilitated learning and hippocampal firing patterns that reflected the commonalities and differences between situations. CA1 and CA3 ensembles revealed differences, but both regions coded new and old information simultaneously. In the SW task, when the environment and task rules were stable but movement direction was reversed, good performance and new place-field maps were established rapidly. CA3 codes integrated the STD and SW situations most powerfully, in that most CA3 neurons had a place field in the same arm in the two tasks even as precise rate maps and firing rates were uncorrelated. In contrast, the overall population of CA1 cells distinguished the new and old situations with strongly anticorrelated firing patterns, and the degree of anticorrelation declined during memory errors. In the ALT task, when the same procedural rules were maintained but the environmental stimuli were unfamiliar, performance was initially impaired, CA3 place fields were absent, CA1 fields were unstable, and their population vectors were anticorrelated. As performance improved, new CA1 place-field maps stabilized and more often occupied the same arms as in the STD while CA3 fields remained silent. Thus, when prior knowledge facilitated learning, CA1 and CA3 place fields revealed different patterns of partial rate and global remapping that simultaneously encoded the similarities and differences between new and familiar tasks.

\section{The SW task}

Performance transferred almost immediately between the STD and SW tasks. Many CA3 place fields were active in the same arms in the STD and SW (Figs. 2b,c, 3b), suggesting that CA3 activity reflected a transfer or generalization of common information across tasks, but neither precise place-field maps nor firing rates were maintained (Fig. 4b,d) (Leutgeb et al., 2004). CA3 thereby signaled different information across spatial scales. At the scale of an entire maze arm, the functional discriminandum in the plus maze, place-field categories revealed stable signals. The mechanisms that "anchored" some CA3 place fields in the same arms while precise location and firing-rates changed are unknown. The STD and SW tasks included identical local and distal cues, but altered movement sequences. The different sequences would change the relationships between cues and actions, disrupting predictions based on the expected outcome of self-movement. Different neuronal signals may have contributed to the partial remapping that we observed (Nakazawa et al., 2002; Lee et al., 2004; Vazdarjanova and Guzowski, 2004; Gold and Kesner, 2005;
Nakashiba et al., 2008). The cognitive map theory suggests that the hippocampus encodes general locations based on distal cues, and precise locations based on local and idiothetic cues (O'Keefe and Nadel, 1978; Knierim and Rao, 2003). From this view, entorhinal input to CA3 may have provided a general spatial signal that maintained activity in the same maze arm. Simultaneously, dentate input to CA3 may have conveyed on-line information (Lisman, 1999), which with recurrent inputs (Nakazawa et al., 2002) may have influenced the precise firing location and rate within arms (McHugh et al., 2007).

CA1 population vectors were anticorrelated between the STD and SW (Fig. 5a), and the degree of anticorrelation predicted SW performance. The anticorrelation occurred because most CA1 cells were active in nonoverlapping places in the two tasks (Fig. $2 a$ ), not because place fields accumulated near the "switched" goal locations (Hollup et al., 2001; Fyhn et al., 2002). The distinct patterns of CA1 activity may have reduced memory interference when identical places were associated with different behavioral sequences, as suggested previously. In an olfactory-delayed nonmatch to sample task, CA1 activity reflected a comparison between odors presented in the same places (Otto and Eichenbaum, 1992). In a similar operant task, hippocampal population codes were uncorrelated during correct and error trials (Deadwyler et al., 1996). Though the anticorrelated CA1 activity between STD and SW tasks are certainly related to movement direction (McNaughton et al., 1983; Muller and Kubie, 1987; Markus et al., 1995; Gothard et al., 1996), the causal relationship between placefield directionality and memory is unclear. Here, the stability of CA3 place fields, and the positive correlation between the CA1 discriminative activity and performance suggests that memory demands contributed significantly to coding dynamics (Alvernhe et al., 2011). Further experiments that dissociate the influence of these variables should determine whether they are computed within the hippocampus or in cortical circuits (Hasselmo and Giocomo, 2006).

\section{The ALT task}

Performance was more impaired, and hippocampal coding was more strongly altered in the ALT than the SW task; CA1 fields were destabilized, and most CA3 place fields were silenced (Fig. 10). CA1 population vectors were again anticorrelated compared with the STD, suggesting that CA1 computations may enhance the representational contrast between different situations. In the ALT task, however, the stability of CA1 place fields predicted performance, not anticorrelation with the STD. Across repeated ALT sessions, CA1 place fields settled into more highly correlated, self-consistent patterns (Figs. $7 a, 8 c, 11$ ). In parallel, increasing numbers of CAl cells tended to remain active on the same arm between STD and later ALT sessions (Fig. 7a). These results could indicate that CA1 neurons initially contrasted new and familiar situations and, through repeated comparison, formed associative links between them. Alternatively, both changes may have reflected an increasing influence of common, uncontrolled cues across repeated STD and ALT sessions.

The changes to CA1 place fields during ALT sessions occurred even as CA3 cells were silent in the maze. CA3 place fields were consistently rare throughout the ALT sessions even as the same cells had place fields in the STD sessions recorded before and after ALT trials (Fig. 10). This observation verifies that CA1 dynamics do not require CA3 activity (Nakashiba et al., 2008). It also shows that CA3 activity did not "bridge" the STD and ALT tasks as it appeared to do in the SW task, and its absence may have contributed in part to the relatively poor transfer of memory perfor- 
mance in the ALT. Memory performance correlates with CA1 place-field stability (Kentros et al., 2004), which is independent of CA3 input (Mizumori et al., 1989; Brun et al., 2002; Nakashiba et al., 2008). Future experiments should determine why CA3 place fields were silent and determine whether the unfamiliar cues provided insufficient excitatory input to CA3 from extrinsic or recurrent pathways, or increased inhibition by local inhibitory interneurons (Freund and Buzsáki, 1996; Acsády et al., 1998; Mori et al., 2007).

\section{CA1 and CA3}

The behavioral correlates of CA1 and CA3 activity differed, verifying that hippocampal processing does not simply follow a sequential "trisynaptic circuit" from the entorhinal cortex to CA1 via the dentate gyrus and CA3. CA1 and CA3 have parallel inputs, distinct network architectures, and diverging output pathways (Amaral and Witter, 1989; Cenquizca and Swanson, 2007), and provide different computations that independently inform behavior (Leutgeb et al., 2004). CA1 place fields do not require input from the dentate gyrus, CA3, or the entorhinal cortex (McNaughton et al., 1989; Van Cauter et al., 2008; compared with Miller and Best, 1980; Brun et al., 2002), and CA1 and CA3 place fields can be manipulated independently (Mizumori et al., 1989; Martig and Mizumori, 2011).

We propose that two key differences between CA1 and CA3 networks account for their distinct coding properties. (1) In contrast to CA3, CA1 receives powerful excitatory signals from the prefrontal cortex via the nucleus reunions (Vertes, 2006), and important dopaminergic input from the VTA. These inputs could support CA1 place fields despite the silencing of CA3 cells in the ALT task. (2) Most excitatory synapses on CA3 neurons are from other CA3 cells, and this recurrent architecture of CA3 has long been proposed to support "pattern completion" through attractor dynamics (Marr, 1971; McNaughton and Morris, 1987). The recurrent network in CA3 could maintain arm coding in the STD and SW tasks by conveying signals about familiar stimuli to overlapping groups of active cells. In unfamiliar environments, however, associations among stimuli coded by collateral synapses should be weak, reducing the net excitatory input and silencing CA3 cells in the ALT task.

\section{Global and rate remapping}

Groups of CA3 cells tend to respond relatively coherently when environmental or behavioral variables are changed (e.g., wall color or movement direction). In contrast, CAl cells typically respond discordantly to the same manipulations, so that subsets of CA1 neurons respond simultaneously to different relationships among environmental and behavioral factors (Tanila et al., 1997; Knierim, 2002). One interpretation of these data suggests that: (1) CA3 ensembles represented one spatial coordinate system in each environment and code different situations by modulating firing rates within a stable population of cells (partial rate remapping) (Leutgeb et al., 2005; Leutgeb and Leutgeb, 2007); (2) CA1 ensembles code arbitrary similarities and differences among situations through partial global remapping (Leutgeb et al., 2004); (3) across environments the active population of CA3 cells should be entirely distinct while subsets of CA1 cells should reflect features common to both environments. The present results verified that across tasks in the same familiar environment, CA 3 codes were more stable than CA1, and were twice as likely to have fields in the same arm. Furthermore, in an unfamiliar environment, CA3 coding changed dramatically: few cells had place fields, and those that did fired in anticorrelated patterns between tasks. In contrast, CA1 activity was maintained at normal levels, and the cells were more likely to have fields in the same arm across tasks than CA3. These observations confirm previous findings that CA3 cells have nonlinear responses that tend toward either pattern completion or separation (Guzowski et al., 2004). The results do not suggest, however, that the tendency toward partial global and rate remapping distinguishes $\mathrm{CA} 1$ from $\mathrm{CA} 3$ coding. Subsets of both CA1 and CA 3 cells responded with distinct firing rates and spatial distributions across tasks, and partial rate and global remapping occurred simultaneously in both cell fields. These observations probably reflect task demands, which strongly influence hippocampal coding in otherwise identical situations (Smith and Mizumori, 2006; Alvernhe et al., 2008, 2011). Partial remapping suggests a coding hierarchy (Tanila et al., 1997) that simultaneously bridges familiar items (arms) while discriminating different behaviors with respect to those items (e.g., sequences of precise locations within the arm). Such a coding hierarchy may contribute to memory-guided learning more generally by simultaneously coding the similarities and differences between new and familiar circumstances. In this way, the same computations that allow stored representations to be used flexibly during encoding and retrieval can provide powerful mechanisms for memory-guided learning.

\section{References}

Acsády L, Kamondi A, Sík A, Freund T, Buzsáki G (1998) GABAergic cells are the major postsynaptic targets of mossy fibers in the rat hippocampus. J Neurosci 18:3386-3403.

Alvernhe A, Van Cauter T, Save E, Poucet B (2008) Different CA1 and CA3 representations of novel routes in a shortcut situation. J Neurosci 28:7324-7333.

Alvernhe A, Save E, Poucet B (2011) Local remapping of place cell firing in the Tolman detour task. Eur J Neurosci 33:1696-1705.

Amaral DG, Witter MP (1989) The three-dimensional organization of the hippocampal formation: a review of anatomical data. Neuroscience 31:571-591.

Bartlett EC (1932) Remembering. A study in experimental and social psychology. London: Cambridge UP.

Brun VH, Otnass MK, Molden S, Steffenach HA, Witter MP, Moser MB, Moser EI (2002) Place cells and place recognition maintained by direct entorhinal-hippocampal circuitry. Science 296:2243-2246.

Buzsáki G (1986) Hippocampal sharp waves: their origin and significance. Brain Res 398:242-252.

Cenquizca LA, Swanson LW (2007) Spatial organization of direct hippocampal field CA1 axonal projections to the rest of the cerebral cortex. Brain Res Rev 56:1-26.

Deadwyler SA, Bunn T, Hampson RE (1996) Hippocampal ensemble activity during spatial delayed-nonmatch-to-sample performance in rats. J Neurosci 16:354-372.

Fenton AA, Csizmadia G, Muller RU (2000) Conjoint control of hippocampal place cell firing by two visual stimuli. I. The effects of moving the stimuli on firing field positions. J Gen Physiol 116:191-209.

Ferbinteanu J, Shapiro ML (2003) Prospective and retrospective memory coding in the hippocampus. Neuron 40:1227-1239.

Freund TF, Buzsáki G (1996) Interneurons of the hippocampus. Hippocampus 6:347-470.

Fyhn M, Molden S, Hollup S, Moser MB, Moser E (2002) Hippocampal neurons responding to first-time dislocation of a target object. Neuron 35:555-566.

Gold AE, Kesner RP (2005) The role of the CA3 subregion of the dorsal hippocampus in spatial pattern completion in the rat. Hippocampus 15:808-814.

Gothard KM, Skaggs WE, McNaughton BL (1996) Dynamics of mismatch correction in the hippocampal ensemble code for space: interaction between path integration and environmental cues. J Neurosci 16:8027-8040.

Guzowski JF, Knierim JJ, Moser EI (2004) Ensemble dynamics of hippocampal regions CA3 and CA1. Neuron 44:581-584.

Harlow HF (1949) The formation of learning sets. Psychol Rev 56:51-65. 
Hasselmo ME, Giocomo LM (2006) Cholinergic modulation of cortical function. J Mol Neurosci 30:133-135.

Hollup SA, Molden S, Donnett JG, Moser MB, Moser EI (2001) Accumulation of hippocampal place fields at the goal location in an annular watermaze task. J Neurosci 21:1635-1644.

Jeffery KJ, Gilbert A, Burton S, Strudwick A (2003) Preserved performance in a hippocampal-dependent spatial task despite complete place cell remapping. Hippocampus 13:175-189.

Karlsson MP, Frank LM (2008) Network dynamics underlying the formation of sparse, informative representations in the hippocampus. J Neurosci 28:14271-14281.

Kennedy PJ, Shapiro ML (2009) Motivational states activate distinct hippocampal representations to guide goal-directed behaviors. Proc Natl Acad Sci U S A 106:10805-10810.

Kentros CG, Agnihotri NT, Streater S, Hawkins RD, Kandel ER (2004) Increased attention to spatial context increases both place field stability and spatial memory. Neuron 42:283-295.

Kentros C, Hargreaves E, Hawkins RD, Kandel ER, Shapiro M, Muller RV (1998) Abolition of long-term stability of new hippocampal place cell maps by NMDA receptor blockade. Science 280:2121-2126.

Knierim JJ (2002) Dynamic interactions between local surface cues, distal landmarks, and intrinsic circuitry in hippocampal place cells. J Neurosci 22:6254-6264.

Knierim JJ, Rao G (2003) Distal landmarks and hippocampal place cells: effects of relative translation versus rotation. Hippocampus 13:604-617.

Lee I, Yoganarasimha D, Rao G, Knierim JJ (2004) Comparison of population coherence of place cells in hippocampal subfields CA1 and CA3. Nature 430:456-459.

Leutgeb S, Leutgeb JK (2007) Pattern separation, pattern completion, and new neuronal codes within a continuous CA3 map. Learn Mem 14:745-757.

Leutgeb S, Leutgeb JK, Treves A, Moser MB, Moser EI (2004) Distinct ensemble codes in hippocampal areas CA3 and CA1. Science 305:12951298.

Leutgeb S, Leutgeb JK, Barnes CA, Moser EI, McNaughton BL, Moser MB (2005) Independent codes for spatial and episodic memory in hippocampal neuronal ensembles. Science 309:619-623.

Lisman JE (1999) Relating hippocampal circuitry to function: recall of memory sequences by reciprocal dentate-CA3 interactions. Neuron 22:233-242.

Markus EJ, Qin YL, Leonard B, Skaggs WE, McNaughton BL, Barnes CA (1995) Interactions between location and task affect the spatial and directional firing of hippocampal neurons. J Neurosci 15:7079-7094.

Marr D (1971) Simple memory: a theory for archicortex. Philos Trans R Soc Lond B Biol Sci 262:23-81.

Martig AK, Mizumori SJ (2011) Ventral tegmental area disruption selectively affects CA1/CA2 but not CA3 place fields during a differential reward working memory task. Hippocampus 21:172-184.

McHugh TJ, Jones MW, Quinn JJ, Balthasar N, Coppari R, Elmquist JK, Lowell BB, Fanselow MS, Wilson MA, Tonegawa S (2007) Dentate gyrus NMDA receptors mediate rapid pattern separation in the hippocampal network. Science 317:94-99.

McNaughton BL, Morris RGM (1987) Hippocampal synaptic enhancement and information storage within a distributed memory system. Trends Neurosci 10:408-415.

McNaughton BL, Barnes CA, O'Keefe J (1983) The contributions of position, direction, and velocity to single unit activity in the hippocampus of freely-moving rats. Exp Brain Res 52:41-49.

McNaughton BL, Barnes CA, Meltzer J, Sutherland RJ (1989) Hippocampal granule cells are necessary for normal spatial learning but not for spatially-selective pyramidal cell discharge. Exp Brain Res 76:485-496.

Miller VM, Best PJ (1980) Spatial correlates of hippocampal unit activity are altered by lesions of the fornix and endorhinal cortex. Brain Res 194:311-323.

Mizumori SJ, McNaughton BL, Barnes CA, Fox KB (1989) Preserved spatial coding in hippocampal CA1 pyramidal cells during reversible suppression of CA3c output: evidence for pattern completion in hippocampus. J Neurosci 9:3915-3928.

Mori M, Gähwiler BH, Gerber U (2007) Recruitment of an inhibitory hippocampal network after bursting in a single granule cell. Proc Natl Acad Sci U S A 104:7640-7645.

Muller RU, Kubie JL (1987) The effects of changes in the environment on the spatial firing of hippocampal complex-spike cells. J Neurosci 7:1951-1968.

Muller RU, Kubie JL, Ranck JB Jr (1987) Spatial firing patterns of hippocampal complex-spike cells in a fixed environment. J Neurosci 7:1935-1950.

Nakashiba T, Young JZ, McHugh TJ, Buhl DL, Tonegawa S (2008) Transgenic inhibition of synaptic transmission reveals role of CA3 output in hippocampal learning. Science 319:1260-1264.

Nakazawa K, Quirk MC, Chitwood RA, Watanabe M, Yeckel MF, Sun LD, Kato A, Carr CA, Johnston D, Wilson MA, Tonegawa S (2002) Requirement for hippocampal CA3 NMDA receptors in associative memory recall. Science 297:211-218.

O’Keefe J, Nadel L (1978) The hippocampus as a cognitive map. Oxford, UK: Oxford UP.

Otto T, Eichenbaum H (1992) Neuronal activity in the hippocampus during delayed non-match to sample performance in rats: evidence for hippocampal processing in recognition memory. Hippocampus 2:323-334.

Shapiro ML, Tanila H, Eichenbaum H (1997) Cues that hippocampal place cells encode: dynamic and hierarchical representation of local and distal stimuli. Hippocampus 7:624-642.

Smith DM, Mizumori SJ (2006) Learning-related development of contextspecific neuronal responses to places and events: the hippocampal role in context processing. J Neurosci 26:3154-3163.

Tanila H, Shapiro ML, Eichenbaum H (1997) Discordance of spatial representation in ensembles of hippocampal place cells. Hippocampus 7:613-623.

Thompson LT, Best PJ (1990) Long-term stability of the place-field activity of single units recorded from the dorsal hippocampus of freely behaving rats. Brain Res 509:299-308.

Thorndike EL (1919) The influence of improvement in one mental function upon the efficiency of other functions. In: Educational psychology: briefer course, pp 259-282. New York: Columbia UP.

Van Cauter T, Poucet B, Save E (2008) Unstable CA1 place cell representation in rats with entorhinal cortex lesions. Eur J Neurosci 27:1933-1946.

Vazdarjanova A, Guzowski JF (2004) Differences in hippocampal neuronal population responses to modifications of an environmental context: evidence for distinct, yet complementary, functions of CA3 and CA1 ensembles. J Neurosci 24:6489-6496.

Vertes RP (2006) Interactions among the medial prefrontal cortex, hippocampus and midline thalamus in emotional and cognitive processing in the rat. Neuroscience 142:1-20.

Wilson MA, McNaughton BL (1993) Dynamics of the hippocampal ensemble code for space. Science 261:1055-1058.

Witter MP (2007) Intrinsic and extrinsic wiring of CA3: indications for connectional heterogeneity. Learn Mem 14:705-713. 